\title{
BINOMIAL-COEFFICIENT MULTIPLES OF IRRATIONALS
}

\author{
Terrence M. Adams and Karl E. Petersen
}

\begin{abstract}
Denote by $x$ a random infinite path in the graph of Pascal's triangle (left and right turns are selected independently with fixed probabilities) and by $d_{n}(x)$ the binomial coefficient at the $n$ 'th level along the path $x$. Then for a dense $G_{\delta}$ set of $\theta$ in the unit interval, $\left\{d_{n}(x) \theta\right\}$ is almost surely dense but not uniformly distributed modulo 1 .
\end{abstract}

\section{INTRODUCTION}

The Pascal graph is the directed infinite planar graph with vertices $(n, k)$, for $n=0,1, \ldots$ and $k=0, \ldots, n$ and two edges coming out of each vertex $(n, k)$, one to $(n+1, k)$ and one to $(n+1, k+1)$. Labeling edges of the first kind by 0 and of the second kind by 1 produces a natural correspondence between infinite sequences $x \in \Omega=\{0,1\}^{\mathbb{N}}$ and infinite paths in the Pascal graph which start at the root vertex $(0,0)$. We will denote by $d_{n}(x)$ the binomial coefficient $C\left(n, k_{n}(x)\right)$ found at the $n$ 'th vertex of $x$, if the Pascal graph is superimposed on the Pascal triangle. The Pascal adic transformation on the space $X$ of infinite paths (see $[9,11,12,6]$ ) corresponds to the map $T: \Omega \rightarrow \Omega$ given by $T\left(1^{p} 0^{q} 01 \ldots\right)=0^{q} 1^{p} 10 \ldots$ for $p, q \geq 0$. Vershik [9] noted that the invariant ergodic measures for this map are exactly the Bernoulli measures $\mu_{\alpha}=\mathcal{B}(\alpha, 1-\alpha)$ on $\Omega$ and conjectured [10] that they are weakly mixing. It was noted in [6] that if, for a fixed Bernoulli measure on $\Omega, \lambda$ is an eigenvalue of $T$, then $\lambda^{d_{n}(x)} \rightarrow 1$ for a.e. $x$. The question of whether or not there exist such $\lambda$, and its variants concerning the distribution of the points $\lambda^{d_{n}(x)}$ on the unit circle for typical $x$ or indeed for all $x$, some of them also mentioned in [6], lead to the study of the distribution modulo 1 of binomial-coefficient multiples of irrationals; answering many of these questions seems to demand deeper understanding of the divisibility properties of binomial coefficients than we have at present.

While we are not yet able to answer the question of weak mixing for the Pascal adic transformation, we do have some progress on related questions. First, we note that if $x$ is a path in the Pascal graph which tracks a line of a fixed slope $\alpha$, then the proportion of $j$ 's, $0 \leq j \leq n$, for which $d_{j}(x)$ is divisible by a fixed prime $q$ tends to 1 as $n \rightarrow \infty$. Using this, we construct an uncountable set of $\lambda$ on the unit circle such that for a.e. path $x$ in the Pascal graph (with respect to a fixed Bernoulli measure) the points $\lambda^{d_{n}(x)}$ are not uniformly distributed on the circle, since asymptotically too large a fraction of them are near 1 . Thus these points might be candidates for eigenvalues of $T$ - but we construct such $\lambda$ for which the $\lambda^{d_{n}(x)}$ are dense. We also list several further observations, questions, and conjectures about the distribution of these points; perhaps the strongest conjecture (also mentioned

1991 Mathematics Subject Classification. Primary 28D05, 28D99..

Key words and phrases. uniform distribution modulo 1, Pascal adic transformation, measure- 
in [6]) is the following: If $\lambda \in \mathbb{C}$ and there exists a path $x$ in the Pascal graph for which $\lambda^{d_{n}(x)} \rightarrow 1$, then $\lambda=1$.

The second author gratefully acknowledges the support of the Erwin Schrödinger Institute, Vienna, where part of this research was conducted.

\section{Intersections OF LINES With SiERPINSKI'S GASKET}

Let $E$ denote the triangle with vertices $(0,0),(1,0)$, and $(0,1)$. In this section we construct Sierpinski's gasket as a subset of $E$. Then we prove that every straight line path (with slope strictly greater than 0) through Sierpinski's gasket intersects the gasket in a set with one-dimensional Lebesgue measure 0. (Since Sierpinski's gasket has two-dimensional Lebesgue measure 0, Fubini's theorem guarantees that almost every line with a specified slope intersects Sierpinski's gasket with one-dimensional Lebesgue measure 0, but this is not sufficient for our purpose.) We give two lemmas which set up the general proof. Also we apply this to the generalized Sierpinski gasket defined at the end of this section.

Given a natural number $n$, let $E_{n}^{1}$ denote the interior of the right triangle with vertices $\left(\left(2^{n-1}-1\right) / 2^{n-1}, 1 / 2^{n}\right),\left(\left(2^{n}-1\right) / 2^{n}, 0\right)$ and $\left(\left(2^{n}-1\right) / 2^{n}, 1 / 2^{n}\right)$ and let $E_{n}^{2}$ be the interior of the triangle with vertices $\left(1 / 2^{n},\left(2^{n-1}-1\right) / 2^{n-1}\right),\left(0,\left(2^{n}-1\right) / 2^{n}\right)$ and $\left(1 / 2^{n},\left(2^{n}-1\right) / 2^{n}\right)$. We view the collection of $E_{n}^{i}$ 's contained in the larger triangle $E$ with vertices $(0,0),(1,0)$ and $(0,1)$. The next two lemmas concern the intersection of the $E_{n}^{i}$ 's with a straight line of slope $\gamma>0$ (where $\gamma$ corresponds to the invariant Bernoulli measure $\mu_{\alpha}$, with $\alpha=1 /(1+\gamma)$ for the Pascal adic transformation).

Lemma 2.3. Given $\alpha>0$ there exists $\eta=\eta(\alpha)>0$ such that: for any straight line $L$ of slope $\alpha$ which intersects the interior of $E$, there exists $n$ and $i$ such that

$$
\frac{\mu\left(L \cap E_{n}^{i}\right)}{\mu(L \cap E)} \geq \eta .
$$

Before we prove Lemma 2.3 we state and prove the preliminary Lemma 2.2.

Lemma 2.2. Given $\gamma>0$ there exist positive real numbers $\epsilon=\epsilon(\gamma)$ and $\eta=\eta(\gamma)$ such that for all $b \in[-\epsilon,(1-\epsilon) / 2]$ and $L_{b}=\{(x, \gamma(x-b)): x \in \mathbb{R}\}$ we have

$$
\frac{\mu\left(L_{b} \cap E_{1}\right)}{\mu\left(L_{b} \cap E\right)} \geq \eta
$$

Proof. First we find the intersection of $L_{b}$ with the hypotenuse of $E_{1}$. Solving $y=\gamma(x-b)$ and $y=1 / 2-x$ simultaneously, we obtain $x=(1 / 2+\gamma b) /(1+\gamma)$ and $y=\gamma(1 / 2-b) /(1+\gamma)$. Thus if we choose $\epsilon<1 /(2 \gamma)$ our point of intersection will have positive $x$ and $y$ coordinates. Hence the function $f(b)=\mu\left(L_{b} \cap E_{1}\right) / \mu\left(L_{b} \cap E\right)$ is positive and continuous on the closed interval $[-\epsilon,(1-\epsilon) / 2]$ and therefore achieves a positive minimum value $\eta$.

Proof of Lemma 2.3. Any line $L$ which intersects the interior of $E$ intersects either the line segment joining $(0,0)$ to $(1,0)$ or the line segment joining $(0,0)$ to $(0,1)$. Without loss of generality let us consider line segments $L$ intersecting the segment joining $(0,0)$ to $(1,0)$. If we let $I=\{(x, 0): 0 \leq x<1\}$, then $I \cap L \neq \emptyset$. In this 
Choose $\epsilon>0$ as in Lemma 2.2. (In particular $\epsilon<1 /(2 \gamma)$ will work.) We may cover $I$ with subintervals $I_{n}=\left[\left(2^{n-1}-1\right) / 2^{n-1}-\epsilon / 2^{n-1},\left(2^{n}-1\right) / 2^{n}-\epsilon / 2^{n}\right)$; so we have $I=\bigcup_{n=1}^{\infty} I_{n}$. For each $b \in I_{n}$ define $L_{b}=\{(x, \gamma(x-b)): x \in \mathbb{R}\}$ and $f_{n}: I_{n} \rightarrow[0,1]$ as $f_{n}(b)=\mu\left(L_{b} \cap E_{n}\right) / \mu\left(L_{b} \cap E\right)$. The self-similarity properties of the triangles $E_{n}$ imply that each $f_{n}: I_{n} \rightarrow[0,1]$ is continuous, and they all have the same image. Therefore by Lemma 2.2 there exists a single real number $\eta>0$ such that

$$
f_{n}(b)=\frac{\mu\left(L_{b} \cap E_{n}\right)}{\mu\left(L_{b} \cap E\right)} \geq \eta
$$

for all positive integers $n$ and all $b \in I_{n}$.

Now we construct Sierpinski's gasket as a closed nowhere dense subset of $E$. We call triangles in the plane lower triangles if we can label the vertices $\left(a_{1}, b_{1}\right)$, $\left(a_{2}, b_{2}\right)$ and $\left(a_{3}, b_{3}\right)$ so that the right angle is at $\left(a_{2}, b_{2}\right)$ and $a_{2}=\min \left\{a_{1}, a_{3}\right\}$ and $b_{2}=\min \left\{b_{1}, b_{3}\right\}$. Upper triangles have right angle at $\left(a_{2}, b_{2}\right)$ with $a_{2}=\max \left\{a_{1}, a_{3}\right\}$ and $b_{2}=\max \left\{b_{1}, b_{3}\right\}$. Note that given a lower triangle $R$ there is a unique upper triangle (inscribed in $R$ ) whose vertices are the midpoints of the sides of $R$. This upper triangle is denoted $\mathcal{U}(R)$; let $\mathcal{L}(R)=\left\{R_{1}, R_{2}, R_{3}\right\}$ be the collection of lower triangles remaining when we extract $\mathcal{U}(R)$ from $R$. Also given a collection $\mathcal{C}$ of lower triangles let $\mathcal{U}(\mathcal{C})=\{\mathcal{U}(R): R \in \mathcal{C}\}$ and let

$$
\mathcal{L}(\mathcal{C})=\bigcup_{R \in \mathcal{C}} \mathcal{L}(R)
$$

We take the triangles in $\mathcal{L}(R)$ to be closed.

The following proposition uses Lemma 2.3 to prove that $\mu(L \cap G)=0$ for any line $L$ with slope $\gamma>0$. First note that Lemmas 2.2 and 2.3 can be extended to any lower triangle playing the role of the initial triangle $E$. Also note that $E_{n}^{i} \in \mathcal{U}\left(\mathcal{L}^{n-1}(E)\right)$ for positive integers $n$ and $i=1,2$. This implies that each $R \in \mathcal{L}^{n}(E)$ is disjoint from the interior of $E_{n}^{i}$. Let

$$
G_{n}=\bigcup_{R \in \mathcal{L}^{n}(E)} R
$$

Then $G=\bigcap_{n=1}^{\infty} G_{n}$ is Sierpinski's gasket.

Proposition 2.4. If $\gamma>0$ and $L=\{(x, \gamma x): x \in \mathbb{R}\}$, then $\mu(G \cap L)=$ $\lim _{n \rightarrow \infty} \mu\left(G_{n} \cap L\right)=0$.

Proof. Choose $\eta=\eta(\gamma)>0$ as in Lemma 2.3. We construct inductively a sequence $n_{j}$ of natural numbers such that for all positive integers $j$ we have

$$
\frac{\mu\left(L \cap G_{n_{j}}\right)}{\mu(L \cap E)} \leq(1-\eta)^{j} .
$$

For the primary case Lemma 2.3 ensures that there exists $E_{n_{1}}^{i_{1}} \in \mathcal{U}\left(\mathcal{L}^{n_{1}-1}(E)\right)$ such that $\mu\left(L \cap E_{n_{1}}^{i_{1}}\right) / \mu(L \cap E) \geq \eta$. (Actually $n_{1}=1$.) Hence $\mu\left(L \cap G_{n_{1}}\right) / \mu(L \cap E) \leq$ $1-\eta$.

For the general case suppose that $n_{k}$ satisfies $\mu\left(L \cap G_{n_{k}}\right) / \mu(L \cap E) \leq(1-\eta)^{k}$. 
$\mathcal{M}=\left\{m: 1 \leq m \leq p, \quad R_{m} \cap L \neq \emptyset\right\}$. Thus $\mu\left(R_{m} \cap L\right)=0$ for $m \notin \mathcal{M}$. For each $m \in$ $\mathcal{M}$, by Lemma 2.3 there exists a positive integer $e(m)$ and $E(m) \in \mathcal{U}\left(\mathcal{L}^{e(m)-1}\left(R_{m}\right)\right)$ such that $\mu(L \cap E(m)) / \mu\left(L \cap R_{m}\right) \geq \eta$. Let $e=\max _{m \in \mathcal{M}}\{e(m)\}$, and let $n_{k+1}=$ $n_{k}+e$. Therefore we have

$$
\begin{aligned}
\mu\left(L \cap G_{n_{k+1}}\right) & \leq(1-\eta) \mu\left(L \cap G_{n_{k}}\right) \\
& \leq(1-\eta)^{k+1} \cdot \square
\end{aligned}
$$

Now we define the generalized Sierpinski gasket and give the analogous proposition which may be proved by the same method. Let $E$ be the closed triangle described above. Given a positive integer $q$ the lines $y=p / q, x=p / q$ and $y=p / q-x$ partition $E$ into $q^{2}$ triangles with $q(q-1) / 2$ upper triangles and $q(q+1) / 2$ lower triangles. Let $\mathcal{U}_{q}(E)$ be the collection of upper triangles and $\mathcal{L}_{q}(E)$ be the collection lower triangles. (Take the members of $\mathcal{U}_{q}(E)$ to be open and the members of $\mathcal{L}_{q}(E)$ to be closed.) Define $\mathcal{U}_{q}(\mathcal{C})$ and $\mathcal{L}_{q}(\mathcal{C})$ analogously for any collection $\mathcal{C}$ of lower triangles. We obtain

$$
G_{q}^{n}=\bigcup_{R \in \mathcal{L}_{q}^{n}(E)} R, \quad G_{q}=\bigcap_{n=1}^{\infty} G_{q}^{n} .
$$

Proposition 2.5. If $\gamma>0$ and $L=\{(x, \gamma x): x \in \mathbb{R}\}$, then for all positive integers $q$

$$
\mu\left(G_{q} \cap L\right)=\lim _{n \rightarrow \infty} \mu\left(G_{q}^{n} \cap L\right)=0 .
$$

Proof. We explain how to choose sets $E_{n}^{i}(q)$ analogous to the $E_{n}^{i}$ above. Then the analogues of the previous lemmas and proposition follow in the same manner as before.

Given a lower triangle $R$ let $\mathcal{L}_{q}(R)$ be the collection of two lower triangles: the top left, lower triangle from $\mathcal{L}_{q}(R)$ and the bottom right, lower triangle from $\mathcal{L}_{q}(R)$. Similarly we define $\mathcal{L}_{q}^{n}(\mathcal{C})$. The sets $E_{n}^{i}(q)$ are chosen in $\mathcal{U}_{q}\left(\mathcal{L}_{q}^{n-1}(E)\right)$.

\section{Binomial coefficients modulo a prime}

ALONG A RANDOM PATH IN PASCAL'S TRIANGLE

If Pascal's triangle is reduced modulo a prime $q$, a well-known self-similar pattern (which can be produced by a cellular automaton) results; this is a consequence of Kummer's Carry Theorem [4] and the resulting formula of Lucas [5]. The parts of the triangle that correspond to the upper triangles (which form $\left(G_{q}\right)^{c}$ ) (the 'voids') consist of regions in which the binomial coefficients are divisible by $q$. The following theorem says that since a line of slope $\gamma$ spends most of its time outside of each $G_{q}^{n}$, a $\mu_{\alpha}$-typical path $x$, which eventually approaches a line of slope $\gamma=(1-\alpha) / \alpha$ in Pascal's triangle, spends most of its time on vertices which carry binomial coefficients divisible by $q$.

Theorem 3.1. If $q$ is prime and $0<\alpha<1$, then for $\mu_{\alpha}$-almost all $x$ we have

$$
\lim _{n \rightarrow \infty} \frac{1}{n} \sum^{n-1}\left|e^{2 \pi i d_{j}(x) / q}-1\right|=0
$$


Proof. Let $\epsilon>0$. Using Proposition 2.5, choose $N$ so large that if $n \geq N$ then $\mu\left(G_{q}^{n} \cap L_{\alpha}\right)<\epsilon$. Notice that since the complement of $G_{q}^{N}$ is a union of finitely many triangles, if we move $L_{\alpha}$ just a small amount we cannot decrease the Lebesgue measure of its intersection with $\left(G_{q}^{N}\right)^{c}$ by very much. Thus we may choose $\delta>0$ and then a large-enough natural number $M$ such that if the part within our unit triangle $E$ of the band of width $\delta$ about the line $L_{\alpha}$ is cut into $M$ equally-spaced chunks by lines parallel to the hypotenuse of $E$, and if one point is chosen from each of those chunks, then the proportion of those points which are in $G_{q}^{n}$ is still less than $2 \epsilon$.

Let $S_{k}(x)$ denote the number of 1's along the path $x$ (regarded as a sequence in $\left.\Omega=\{0,1\}^{\mathbb{Z}}\right)$. By the Ergodic Theorem, for $\mu_{\alpha}$-almost every $x$ there is $K=K(x)$ such that

$$
\left|S_{k}(x)-k \alpha\right|<k \delta \quad \text { for all } k \geq K .
$$

Choose a time $M>K / \delta$, and consider Pascal's triangle down to that level, including subtriangles of rank up to $N$. When this part of Pascal's triangle is scaled down to lie over our unit triangle $E$, we see the subtriangles that form $\left(G_{q}^{N}\right)^{c}$, and the scaled-down path $x$ lies entirely inside the band of width $\delta$ about $L_{\gamma}$, the line of slope $\gamma=(1-\alpha) / \alpha$ in the Sierpinski gasket. We have arranged that the proportion of vertices of the scaled-down path which are in $\left(G_{q}^{N}\right)^{c}$ is at least $1-2 \epsilon$, and hence the proportion of vertices of the path $x$ at which the binomial coefficients $d_{j}(x)$ are divisible by $q$ is at least $1-2 \epsilon$.

\section{Main Result: A CONSTRUCtion of SPECial iRRATiOnals}

In this section we use Theorem 3.1 to construct an uncountable dense set (in fact a $\left.G_{\delta}\right) \Lambda \subset[0,1)$ such that for each $\theta \in \Lambda$ and for $\mu_{\alpha}$-almost every $x \in X$ we have that the sequence $\left\{d_{j}(x) \theta\right\}$ is not uniformly distributed modulo 1 . In particular we obtain a result similar to Theorem 3.1, but with the limit replaced by liminf.

Theorem 4.1. There exist a dense $G_{\delta}$ set $\Lambda \subset[0,1)$ and a set of full $\mu_{\alpha}$-measure $Y \subset X$ so that for each $\theta \in \Lambda$ and $x \in Y$ we have

$$
\liminf _{n \rightarrow \infty} \frac{1}{n} \sum_{j=0}^{n-1}\left|e^{2 \pi i d_{j}(x) \theta}-1\right|=0
$$

Proof. Let $\left\{\epsilon_{n}\right\}$ be a sequence of positive real numbers satisfying $\sum_{n=1}^{\infty} \epsilon_{n}<\infty$, and let $\left\{q_{n}\right\}$ be a sequence of primes increasing to $\infty$. We will produce sequences $R_{n}$ of natural numbers and $\delta_{n}>0$ so that if

$$
\begin{gathered}
Y_{n}=\left\{x \in X: \frac{1}{R_{n}} \sum_{j=0}^{R_{n}-1}\left|e^{2 \pi i d_{j}(x) p / q_{n}}-1\right|<\frac{1}{n} \text { for } p=0,1, \ldots, q_{n}-1\right\} \\
\Lambda_{n}=\left\{\theta \in[0,1): \text { there exists } p=0,1, \ldots, q_{n}-1 \text { with }\left|\theta-\frac{p}{q_{n}}\right|<\delta_{n}\right\}, \\
\Lambda=\bigcap^{\infty} \bigcup^{\infty} \Lambda_{n}, \text { and } Y=\bigcup^{\infty} \bigcap^{\infty} Y_{n},
\end{gathered}
$$


then

$$
\liminf _{n \rightarrow \infty} \frac{1}{n} \sum_{j=0}^{n-1}\left|e^{2 \pi i d_{j}(x) \theta}-1\right|=0
$$

for all $\theta \in \Lambda$ and all $x \in Y$.

For each $n$, by Theorem 3.1 we may choose $R_{n}$ so that $\mu_{\alpha}\left(Y_{n}\right)>1-\epsilon_{n}$. Then, since

$$
\left\{\frac{1}{R_{n}} \sum_{j=0}^{R_{n}-1}\left|e^{2 \pi i d_{j}(x) \theta}-1\right|: x \in Y_{n}\right\}
$$

is a finite collection of continuous functions of $\theta$, we may choose $\delta_{n}>0$ so that

$$
\frac{1}{R_{n}} \sum_{j=0}^{R_{n}-1}\left|e^{2 \pi i d_{j}(x) \theta}-1\right|<\frac{2}{n}
$$

for all $x \in Y_{n}$ and all $\theta \in \Lambda_{n}$. Then $\Lambda$ is a dense $G_{\delta}$ with the usual topology, and $\mu_{\alpha}(Y)=1$ because $\sum_{n=1}^{\infty} \epsilon_{n}$ converges.

To verify the outcome of the theorem, first choose $\theta \in \Lambda$ and $x \in Y$. Then there exists a sequence $n_{m} \rightarrow \infty$ such that $\theta \in \Lambda_{n_{m}}$ for all positive integers $m$. Also there exists $k$ such that $x \in Y_{n}$ for $n \geq k$. Hence for $n_{m} \geq k$ we have

$$
\frac{1}{R_{n_{m}}} \sum_{j=0}^{R_{n_{m}}-1}\left|e^{2 \pi i d_{j}(x) \theta}-1\right|<\frac{2}{n_{m}} .
$$

\section{Density Without Uniform Distribution}

In the previous section we constructed a dense $G_{\delta}$ set $\Lambda \subset[0,1)$ such that for each $\theta \in \Lambda,\left\{d_{j}(x) \theta\right\}$ is not uniformly distributed modulo 1 for $\mu_{\alpha}$-almost every $x \in X$. Those $\theta \in \Lambda$, which are irrational, remain candidates for eigenvalues of the Pascal adic transformation. However in this section we will show that if the sequence $\left\{\delta_{n}\right\}$ converges to zero sufficiently fast, then $\left\{d_{j}(x) \theta\right\}$ is dense modulo 1 for each $\theta \in \Lambda$ and for $\mu_{\alpha}$-almost every $x \in X$. This excludes these $\theta$ as eigenvalues for the Pascal adic.

We begin by considering Pascal's triangle modulo a prime $q$. Recall that for $n \in \mathbb{N}$ and $1 \leq k \leq q^{n}-1$, we have $C\left(q^{n}, k\right) \cong_{q} 0$. Hence $C\left(q^{n}-1, k\right) \cong_{q}(-1)^{k}$ for $0 \leq k \leq q^{n}-1$, which gives a 'blocking line' on the triangle. It is this 'blocking line' that yields total ergodicity of the Pascal adic. In Lemma 5.1 we note that among the binomial coefficients in the row numbered $q^{n}-2$ one can find all the congruence classes modulo $q$, and in fact they appear in a regular way. This allows us to show that along a random path in the triangle a hit of congruence class $r$ at level $q^{m}$ and of congruence class $p$ at level $q^{n}$ are approximately independent if $m$ and $n$ are far apart, and therefore with probability 1 no congruence class modulo $q$ can be avoided. Consequently, if $\theta$ is very well approximated by rationals $p_{n} / q_{n}$, 
Lemma 5.1. Let $q$ be prime and $n$ a natural number. Then for $k=0, \ldots, q^{n}-2$ we have the following formula:

$$
C\left(q^{n}-2, k\right) \cong_{q}(-1)^{k}(k+1) .
$$

Moreover, for natural numbers $k$ and $p$ satisfying $0 \leq p \leq q-1$ and $0 \leq k \leq$ $q^{n}-2 q-1$ the set

$$
\left\{i: k \leq i \leq k+2 q-1, C\left(q^{n}-2, i\right) \cong_{q} p\right\}
$$

has exactly two elements.

Proof. First we derive the formula inductively. The primary case is trivial: $C\left(q^{n}-\right.$ $2,0)=1=(-1)^{0}(1)$. Assume the formula holds for $k=\ell-1$. Thus for $k=\ell$ we have

$$
\begin{aligned}
C\left(q^{n}-2, \ell\right) & =C\left(q^{n}-1, \ell\right)-C\left(q^{n}-2, \ell-1\right) \\
& \cong_{q}(-1)^{\ell}-(-1)^{\ell-1} \ell \\
& =(-1)^{\ell}[1+\ell] .
\end{aligned}
$$

Now for the second part of the lemma, we note that our formula gives the following:

$$
C\left(q^{n}-2, i+2\right) \cong_{q} \begin{cases}C\left(q^{n}-2, i\right)+2 & \text { if } i \text { even } \\ C\left(q^{n}-2, i\right)-2 & \text { if } i \text { odd } .\end{cases}
$$

Hence if $q=2$ we obtain $C\left(q^{n}-2, k\right) \cong_{q} 1$ for $k$ even and $C\left(q^{n}-2, k\right) \cong_{q} 0$ for $k$ odd. For $q \neq 2$ we have that each of the maps $j \mapsto C\left(q^{n}-2, k+2 j\right) \bmod q$ and $j \mapsto C\left(q^{n}-2, k+1+2 j\right) \bmod q$ gives a bijection of $\{0, \ldots, q-1\}$.

Lemma 5.2. Suppose that for each $n \in \mathbb{N}$, we have a unimodal distribution $f_{n}$ on the set $\left\{0, \ldots, q^{n}-2\right\}$. For each $p$ satisfying $0 \leq p \leq q-1$, let

$$
M_{p}=\left\{m: 0 \leq m \leq q^{n}-2, C\left(q^{n}-2, m\right) \cong_{q} p\right\} .
$$

If $\lim _{n \rightarrow \infty} \max \left\{f_{n}(m): 0 \leq m \leq q^{n}-2\right\}=0$, then

$$
\lim _{n \rightarrow \infty} \sum_{m \in M_{p}} f_{n}(m)=\frac{1}{q}
$$

Proof. We will show that

$$
\lim _{n \rightarrow \infty}\left(\sum_{m \in M_{p}} f_{n}(m)-\sum_{m \in M_{r}} f_{n}(m)\right)=0
$$

for all $p$ and $r$. Without loss of generality, assume that

$$
\sum f_{n}(m) \geq \sum f_{n}(m)
$$


Partition $\left\{0, \ldots, q^{n}-2\right\}$ into subintervals of $2 q$ consecutive numbers with one remaining subinterval of at most $2 q$ consecutive numbers. Discard the subinterval which contains the peak of the distribution $f_{n}$, as well as its two adjacent subintervals, from the set $M_{p}$. Call the remaining set $M_{p}^{*}$. Now for each $m^{*} \in M_{p}^{*}$ there exists $m \in M_{r}$ in the next interval of length $2 q$ towards the peak of $f_{n}$ such that $f_{n}(m) \geq f_{n}\left(m^{*}\right)$. Therefore

$$
\sum_{m \in M_{r}} f_{n}(m) \geq \sum_{m \in M_{p}^{*}} f_{n}(m) \geq \sum_{m \in M_{p}} f_{n}(m)-6 \max \left\{f_{n}(m): 0 \leq m \leq q^{n}-2\right\} .
$$

Let $F_{n}(q, p)$ be the set of paths which pass through a vertex $\left(q^{n}-2, k\right)$ satisfying $C\left(q^{n}-2, k\right) \cong_{q} p$. Lemma 5.2 implies that the conditional probability of the set $F_{n}(q, p)$, given that the path passes through a fixed vertex, converges to $1 / q$ as $n \rightarrow \infty$. Therefore for each $m \in \mathbb{N}$

$$
\lim _{n \rightarrow \infty} \mu_{\alpha}\left(F_{n}(q, p) \cap F_{m}(q, r)\right)=\frac{1}{q} \mu_{\alpha}\left(F_{m}(q, r)\right) .
$$

A standard Hilbert space argument of A. Rényi and P. Révész [8] implies that $F_{n}(q, p), n=1,2, \ldots$ is a mixing sequence of sets. In particular we have Lemma 5.3 , which says $F_{n}(q, p)$ 'sweeps out'. Finally we prove Theorem 5.4 using an approximation technique similar to that used in the previous section.

Lemma 5.3. For $0 \leq p<q$ with $q$ prime and $0<\alpha<1$,

$$
\mu_{\alpha}\left(\bigcup_{n=1}^{\infty} F_{n}(q, p)\right)=1
$$

Theorem 5.4. There exist a dense $G_{\delta}$ set $\Lambda \subset[0,1)$ and a set of full $\mu_{\alpha}$-measure $Y \subset X$ so that for each $\theta \in \Lambda$ and $x \in Y$ the set $\left\{e^{2 \pi i d_{j}(x) \theta}: j \in \mathbb{N}\right\}$ is dense (but not uniformly distributed) in $S^{1}$.

Proof. Let $\left\{\epsilon_{n}\right\}$ be a sequence of positive real numbers satisfying $\sum_{n=1}^{\infty} \epsilon_{n}<\infty$, and let $\left\{q_{n}\right\}$ be a sequence of primes increasing to $\infty$. We will produce sequences $R_{n}$ of natural numbers and $\delta_{n}>0$ so that if

$$
Y_{n}=\bigcap_{p=0}^{q_{n}-1} \bigcup_{j=1}^{R_{n}} F_{j}\left(q_{n}, p\right)
$$

$$
\begin{gathered}
\Lambda_{n}=\left\{\theta \in[0,1): \text { there exists } p_{n}=0,1, \ldots, q_{n}-1 \text { with }\left|\theta-\frac{p_{n}}{q_{n}}\right|<\delta_{n}\right\}, \\
\Lambda=\bigcap_{k=1}^{\infty} \bigcup_{n=k}^{\infty} \Lambda_{n} \text {, and } Y=\bigcup_{k=1}^{\infty} \bigcap_{n=k}^{\infty} Y_{n},
\end{gathered}
$$

then $\left\{e^{2 \pi i d_{j}(x) \theta}: j \in \mathbb{N}\right\}$ is dense but not uniformly distributed for all $\theta \in \Lambda$ and all 
For each $n$, by Lemma 5.3 we may choose $R_{n}$ so that $\mu_{\alpha}\left(Y_{n}\right)>1-\epsilon_{n}$. Then choose

$$
\delta_{n}<\frac{1}{n C\left(q_{n}^{R_{n}}-2,\left(q_{n}^{R_{n}}-2\right) / 2\right)} .
$$

As before, if $\theta \in \Lambda$ and $x \in Y$, then we can find arbitrarily large $n$ such that $\theta \in \Lambda_{n}$ and $x \in Y_{n}$. Now $\theta$ is very well approximated by a rational $p_{n} / q_{n}$, and as $p$ runs through the congruence classes modulo $q_{n}$, the points $p p_{n} / q_{n}$ are $1 / q_{n}$-dense modulo 1. Further, for each congruence class $p$ modulo $q_{n}$ there is $j=1, \ldots, R_{n}$ such that at level $s=q_{n}^{j}$ the path $x$ has its binomial coefficient $d_{s}(x)$ hit that congruence class. Since $\delta_{n}$ has been chosen so small that all the points $d_{s}(x) \theta$ under consideration are very close to the points $d_{s}(x) p_{n} / q_{n}$, and the latter are $1 / q_{n}$-dense, we are done.

\section{Questions And Conjectures}

1. Conjecture $[10,6]$ : For each Bernoulli measure $\mu_{\alpha}$, the Pascal adic transformation $T$ is weakly mixing. This would follow if one could show that $\lambda^{d_{n}(x)} \rightarrow 1$ for a.e. $x$ with respect to $\mu_{\alpha}$ implies $\lambda=1$.

2. Conjecture: If there is a path $x$ such that $\lambda^{d_{n}(x)} \rightarrow 1$, then $\lambda=1$.

3. Does there exist any $\lambda$ in the unit circle such that $\left\{\lambda^{d_{n}(x)}\right\}$ is uniformly distributed in the circle for every $x$ (except for the two paths down the edges)? For such a $\lambda$, the skew-product transformation

$$
S\left(z_{1}, z_{2}, z_{3}, \ldots\right)=\left(\lambda z_{1}, z_{1} z_{2}, z_{2} z_{3}, \ldots\right)
$$

on the infinite torus, known to be uniquely ergodic by results of Weyl [13, 14], Furstenberg [1], Hahn [3], and Postnikov [7], would have the very strong property that we would see a uniformly distributed sequence $\left\{\left(S^{j} z\right)_{k}\right\}$ not only when we looked in a fixed coordinate $k$ at the orbit of a point $z$, but also when we allowed our view to shift one place to the right from time to time: $\left\{\left(S^{j} z\right)_{k_{j}}\right\}$ would be uniformly distributed for each $z$ and each choice of $\left\{k_{j}\right\} \subset \mathbb{N}$ with $k_{j+1}-k_{j} \in\{0,1\}$ for each $j$. (The $\lambda$ that we construct above are at another extreme from this property.)

4. From another theorem of Weyl and Tonelli's Theorem it follows that for almost every $\lambda$ the sequence $\left\{\lambda^{d_{n}(x)}\right\}$ is uniformly distributed for a.e. $x$, with respect to each Bernoulli measure $\mu_{\alpha}$. For which $\lambda$ does this hold? Similarly, what paths $x$ have the property that this sequence is uniformly distributed for each $\lambda$ that is not a root of unity? (By Weyl's Theorem, this is the case for each path $x$ that is eventually diagonal.)

5. Studies like these on divisibility of binomial coefficients by primes suggest questions on simultaneous divisibility by several primes. For example, thinking about the central path in Pascal's triangle and divisibility by 2 and 3 leads to the following Conjecture: The only solutions in nonnegative integers $r$ and distinct $s_{1}, \ldots, s_{m}$ of an equation

$$
2^{r}=3^{s_{1}}+\cdots+3^{s_{m}}
$$

are $1=1,4=1+3$, and $256=1+3+9+243$. We thank Charles Giffen for pointing out that this conjecture was already made by Erdös - see [2]. More generally, if for a prime $p$ we define an integer Cantor set $H(p)$ to consist of all those expansions base $p$ with coefficients in the interval $[0, p / 2]$ (or subject to some other restriction), 


\section{REFERENCES}

1. H. Furstenberg, Strict ergodicity and transformations of the torus, Amer. J. Math. 83 (1961), 573-601.

2. R. K. Guy, Unsolved Problems in Number Theory, Second Edition, Springer-Verlag, New York, 1994, p. 88.

3. F. J. Hahn, On affine transformations of compact abelian groups, Amer. J. Math. 85 (1963), $428-446$.

4. E. E. Kummer, Über die Ergänzungssätze zu den allgemeinen Reciprocitätsgesetzen, J. für Math. 44 (1852), 115-116.

5. E. Lucas, Théorie des fonctions numériques simplement périodiques, Amer. J. Math. 1 (1878), $184-240$.

6. K. Petersen and K. Schmidt, Symmetric Gibbs measures (to appear).

7. A. G. Postnikov, Ergodic problems in the theory of congruences and of Diophantine approximations, Proc. Steklov Inst. Math. 82 (1966), 3-112.

8. A. Rényi, On mixing sequences of sets, Acad. Sci. Hung. 9 (1958), 215-228.

9. A. M. Vershik, Description of invariant measures for actions of some infinite groups, Dokl. Akad. Nauk SSSR 218 (1974), 749-752; Soviet Math. Dokl. 15 (1974), 1396-1400.

10.

11. Uniform algebraic approximation of shift and multiplication operators, Dokl. Akad. Nauk SSSR 259 (1981), 526-529; Soviet Math. Dokl. 24 (1981), 97-100.

12. A. M. Vershik and A. N. Livshitz, Adic models of ergodic transformations, spectral theory, substitutions, and related topics, Adv. Soviet Math. 9 (1992), 185-204.

13. H. Weyl, Über ein Problem aus dem Gebiete der diophantischen Approximationen, Nach. Ges. Wiss. Göttingen (1914), 234-244.

14. H. Weyl, Über die Gleichverteilung von Zahlen mod. Eins, Math. Ann. 77 (1916), 313-352.

Department of Mathematics, CB 3250 Phillips Hall, University of North CarOlina, Chapel Hill, NC 27599-3250, USA

Current address: Department of Mathematics, Ohio State University, Columbus, OH 43210, USA

E-mail address: tadams@math.ohio-state.edu

Department of Mathematics, CB 3250 Phillips Hall, University of North Carolina, Chapel Hill, NC 27599-3250 USA

E-mail address: petersen@math.unc.edu 